\title{
Drastic Improvement in Gas Sensing Characteristics of Phosphorene Nanosheets Under Vacancy Defects and Elemental Functionalization
}

\author{
T. Kaewmaraya, ${ }^{1,2}$ L. Ngamwongwan, ${ }^{1}$ P. Moontragoon, ${ }^{1,2}$ A. Karton ${ }^{3}$ and T. \\ Hussain $^{* 3,4}$ \\ ${ }^{1}$ Integrated Nanotechnology Research Center, Department of Physics, Khon Kaen \\ University, Khon Kaen, Thailand \\ ${ }^{2}$ Nanotec-KKU Center of Excellence on Advanced Nanomaterials for Energy \\ Production and Storage, Khon Kaen, Thailand \\ ${ }^{3}$ School of Molecular Sciences, The University of Western Australia, Perth, WA \\ 6009, Australia
}

${ }^{4}$ Centre for Theoretical and Computational Molecular Science, Australian Institute for Bioengineering and Nanotechnology, The University of Queensland, Brisbane, Qld 4072, Australia

tanveer.hussain@uwa.edu.au

\begin{abstract}
Efficient chemical gas detection is of great importance for various functionalities (such as leakage detection of hazardous and explosive gases in industrial safety systems). The recent discovery of 2-dimensional black phosphorene (BlackP) has created intensive interests towards nano-sensors because of its maximized surface-tovolume ratio and exceptional carrier mobility that potentially deliver the superior performance than the conventional transition-metal oxides sensors. In this work, we have performed first-principles DFT calculations coupled with the statistical analysis to unravel the structural, electronic, and gas sensing characteristics of pristine, defected, and metal substituted BlackP towards toxic $\mathrm{H}_{2} \mathrm{~S}$ and $\mathrm{SO}_{2}$ gas molecules. Our findings have revealed that pristine BlackP weakly interacts with both $\mathrm{H}_{2} \mathrm{~S}$ and $\mathrm{SO}_{2}$ by van der Waals (vdW) forces characterized by the small binding energies. The analysis of electronic properties via the density of states (DOS) indicates that there is a negligible change in DOS after gas exposure, which confirms insensitive sensing. To intensify the binding energies, we have considered defects (mono-vacancy, divacancy, tri-vacancy and quad-vacancy) and substitutional impurities (Ti, Si, Mn and $\mathrm{Fe})$ as the incentives. The presence of mono and di vacancies remains less energetically sensitive to both gas species because of the low adsorption energies.
\end{abstract}


Meanwhile, tri and quad vacancies induce the dissociative adsorption, not suitable for the reversible adsorption-desorption cycles. Substitutional doping by $\mathrm{Fe}$ atoms is found to be a feasible approach to enhance the sensing resolution of $\mathrm{SO}_{2}$ detection because of the remarkable adsorption energy incorporated with the substantial variation in DOS after gas exposure. This modification in electronic properties is facilitated by the charge transfer mechanism from Fe-3 $d$ to $\mathrm{P}-3 p$ which can generate the measurable electrical signal detect by the external circuit of the sensor.

\section{Introduction}

Chemical gas detection has become one of the most crucial issues offering a wide range of applications e.g., leakage detection of hazardous or explosive gases in industrial safety systems, air quality testing and military monitoring) ${ }^{1}$. A chemical gas sensor refers to a device that converts chemical reactions into analytically detectable signals. There are numerous kinds of gas detectors classified by the operational mechanism of the transducers, i. e., semiconductors, oxidation, and catalytic just to name a few. Among them, semiconducting oxides (such as $\mathrm{TiO}_{2}$, $\mathrm{ZnO}$, and $\mathrm{SnO}_{2}$ ) are ubiquitous materials commercially available nowadays because of high sensitivity, low production costs and device scalability. They detect the presence of target analytes by exploiting measurable changes in electrical conductivity (i.e., the local carrier concentration) before and after gas exposure. The change in conductivity is associated with the charge transfer mechanism between the gas molecules and the sensors where the former can act as either donors or acceptors. However, the resolution of semiconducting sensors is generally limited to 1 part per million (ppm) ${ }^{\mathbf{1}}$. This is due to the remarkable noise fluctuation fundamentally originated from thermal motion of charges and defects ${ }^{2}$. Hence, searching for sensing materials offering ultimate sensitivity in the order 1 part per billion (ppb) or even higher remains one of the key challenges in chemical sensing technology.

An innovative approach to attain the ultra-sensitivity is the use of emerging nanomaterials. This is because of the maximized surface-to-volume ratio as exemplified by the extraordinary resolution down to the order of ppb of carbon nanotubes and several kinds of semiconducting nanowires ${ }^{3}, 4$, surpassing the resolution limit of existing semiconducting sensors. Additional interests have also expanded to atomically thin two-dimensional (2D) materials due to the key benefits 
such as the rapid carrier mobility, the unique planar morphology that enlarges the reactive gas-sensing area, superior mechanical stability, and the room-temperature operation $^{5-7}$. These unique features are preferential for practical chemical sensing applications. For instance, recent works have revealed that graphene ${ }^{2}$ and $\mathrm{MoS}_{2}{ }^{\mathbf{8}, 9}$ sensing devices demonstrate the exceptional performance towards various gas species. More recently, a 2D form of black phosphorus, named phosphorene (BlackP), was successfully fabricated ${ }^{\mathbf{1 0 - 1 2}}$. This material shows many appealing properties. It possesses extraordinary carrier mobility (about $1,000 \mathrm{~cm}^{2} \mathrm{~V}^{-1} \mathrm{~s}^{-1}$ at room temperature) 10 ,which intriguingly exceeds that of other 2D semiconductors such as $\mathrm{MoS}_{2}$ (approximately $\left.200 \mathrm{~cm}^{2} \mathrm{~V}^{-1} \mathrm{~s}^{-1}\right)^{13}$. Unlike graphene, BlackP is a direct-gap semiconductor of which the gap size depends on the number of layers ${ }^{14}$. Another feature is that electric and optical properties are anisotropic along the zigzag and the armchair directions due to its puckered wave-like structure with $\mathrm{sp}^{3}$ bonding ${ }^{15}$. This puckered geometry results in the higher surface-to-volume ratio and less out-of-plane electrical conductance than other $2 \mathrm{D}$ materials ${ }^{16,17}$. In particular, the latter gives rise to more sensitive response to the target gas species near BlackP surface ${ }^{\mathbf{1 7}}$. Furthermore, it also shows superior mechanical flexibility ${ }^{\mathbf{1 8}}$. These mentioned features can practically facilitate the chemical sensing efficiency.

The gas-sensing functionality of BlackP for detecting selected important gases has been intensively reported ${ }^{5,17,19,20,21}$. The excellent sensitivity and selectivity of phosphorene for probing $\mathrm{NO}_{2}$ at the concentration of $5 \mathrm{ppb}$ in the dry environment has been experimentally confirmed ${ }^{\mathbf{1 7}}$. On the other hand, it remains less sensitive to other important analytes, particularly sulphur-contained gas species $\left(\mathrm{H}_{2} \mathrm{~S} \text { and } \mathrm{SO}_{2}\right)^{22}$. This is due to weak van der Waals (vdW) interactions between gas molecules and a sensor, which are too weak to be detectable because no electrons are exchanged between gas molecules and phosphorene. The efficient detection of these toxic gases is of crucial importance. Colourless $\mathrm{H}_{2} \mathrm{~S}$ is a highly hazardous gas generally emitted from fuel cell, crude petroleum, volcano and hot springs. Toxic $\mathrm{SO}_{2}$ can be available from manufacturing sulphuric acid and combustion of sulphur. Inhaling these gases with concentration more than 75 ppm (American Conference of Governmental Industrial Hygienists) are drastically harmful to the respiratory system.

A possible way to intensify adsorption energies from weak vdW to chemically 
strong interactions is either to dope impurity atoms or to create defects ${ }^{23-25}$. The explanation is ascribed to the fact that the introduction of suitable dopants yields more electron transfer between gas molecules and the 2D-sensors. Therefore, the objectives of this work are to increase gas-sensing sensitivity of phosphorene by means of introducing defects and impurities. The theoretical approach based on the so-called density functional theory (DFT) and a statistical model will be employed to attain useful information regarding adsorption energies and electronic properties of gasadsorbed phosphorene.

\section{Computational and Modeling details}

Density functional theory (DFT) calculations were performed using VASP $\operatorname{code}^{26}$. The electron-ion interactions were treated by projector-augmented wave $(\mathrm{PAW})^{\mathbf{2 7}}$ approach with the generalized-gradient approximation according to PerdewBurke-Ernzerhof (PBE) formalism ${ }^{28}$. Free-standing phosphorene was modelled by $4 \times 4 \times 1$ supercell consisting of 54 phosphorus atoms and the vacuum spacing of $20 \AA$ was added along the $z$-direction to sufficiently decouple the interaction between periodic replicas. This supercell was based on an orthorhombic unit cell with the optimized lattice constants $a=3.314 \AA$ and $b=4.376 \AA$. The k-point grids for Brillioun zone integration of $9 \times 9 \times 1$ and $17 \times 17 \times 17$ were used for structural optimizations and density of states (DOS) calculations, respectively. A cut-off energy of $500 \mathrm{eV}$ for representing plane wave basis was used throughout the calculations because of being sufficient to attain reasonable energy convergence. The geometrical relaxations were terminated when the Hellman-Feyman force exerting on each atom became less than $10^{-3} \mathrm{eV} / \AA$.

The binding energies of the adsorbed gas molecules on phosphorene monolayer were determined by the following expression

$$
\mathrm{E}_{\mathrm{b}}=\mathrm{E}_{\mathrm{BlackP}}+\text { gas }-\left(\mathrm{E}_{\mathrm{BlackP}}+\mathrm{E}_{\mathrm{gas}}\right)
$$

$\left(\right.$ gas $\left.=\mathrm{H}_{2} \mathrm{~S}, \mathrm{SO}_{2}\right)$

In Eq. (1), the $1^{\text {st }}, 2^{\text {nd }}$ and $3^{\text {rd }}$ terms refer to the total energies of the phosphorene sheet adsorbed by the gas molecules, the corresponding bare phosphorene, and the independent gas molecule, respectively. Moreover, the formation energies of the vacancy defects were determined by the following formalism 


$$
\mathrm{E}_{\mathrm{f}}=\mathrm{E}_{(\text {defected })}-\{(\mathrm{x}-\mathrm{y}) / \mathrm{x}\} \mathrm{E}_{\text {pristine }}
$$

where $E_{f}$ represents the formation energy, $E_{\text {defected }}$ denotes the total energy of defected phosphorene, $\mathrm{E}_{\text {pristine }}$ is the total energies of pristine phosphorene, and $\mathrm{x}$ and $\mathrm{y}$ are the number of atoms in the phosphorene and the number of atoms removed. The calculated adsorption energies presented in this work have incorporated van der Waals interactions using Grimme approach ${ }^{29}$. In addition, the calculations of gas adsorption on to defected systems were spin-polarized to avoid the overestimation of adsorption energy because of the dipole moment-spin interaction ${ }^{\mathbf{2 1}}$. The underlying charge transfer mechanism between the target gas molecule and phosphorene was investigated using Bader analysis ${ }^{\mathbf{3 0}}$.

\section{Results and discussion}

We start with presenting the crystal structures of phosphorene considered in this work. The assembly of structurally relaxed geometries is given in Fig. 1. The optimized pristine phosphorene (the bounding box) has the lattice constants $a=4.58$ $\AA$ and $b=3.32 \AA$, P-P bond lengths of 2.22 and $2.26 \AA$ and P-P-P bond angles of 96.00 and 104.15, being consistent with the previous studies ${ }^{31}$. There are four kinds of neutral vacancies considered in this work namely mono-vacancy (M-vac), di-vacancy (D-vac), tri-vacancy (T-vacancy), and quad-vacancy (Q-vac). The presence of dangling bonds causes notable atomic rearrangements where the $\mathrm{P}$ atoms close to the vacant sites form new bonds with the nearby atoms. There is a drastic variation in the P-P distances, ranging from 2.20 to $2.50 \AA$ as compared to those in the defect-free structure. This structural deformation leads to the reduction in the coordination number and the vacancy sites have consequently become chemically reactive to interacting with foreign gas molecules. In addition, the formation energies associated with M-vac, D-vac, T-vac, and Q-vac are -5.40, -4.46, -4.48, and -5.03 eV, respectively. Apparently, the formation of M-vac and T-vac are energetically more preferable than D-vac ${ }^{32}$. It is important to mention here that the formation energy of phosphorene, especially in case of M-vac, is much smaller than that of the most studied 2D system, graphene. ${ }^{33}$

The expected increase in chemical reactivity of BlackP with defects and impurities to gas molecules (here $\mathrm{H}_{2} \mathrm{~S}$ and $\mathrm{SO}_{2}$ ) was exhaustively examined by searching for the lowest-energy structures. Numerous initial configurations of 
individual gas species were examined and the corresponding adsorption energies after structural optimization were evaluated. In principle, the gas-sensing mechanism depends on the measurable change in the electrical conductivity of a sensor after exposing it to the target gas species. The adsorption of gas molecules can either increase or decrease the conductivity, depending on the variation in carrier concentration induced by the charge transfer between the target molecules and the sensor (i. e. the adsorbate acting as either a donor or an acceptor). The transfer is facilitated by the difference in the electronegativity. This fundamental mechanism coupled with chemical bonding is theoretically reflected by the adsorption energy. A strong adsorption energy represents a strong chemical bond where the charge transfer process takes place and it consequently induces the detectable contrast in the electrical resistivity. Hence, the contrast in conductivity therefore defines the sensitivity. On the other hand, the low adsorption energy indicates a weak vdW bond, which does not yield the marked change in resistivity. The appropriate adsorption energy should be around $1.00 \mathrm{eV}$, which represents weak chemical interaction and it also facilitates the complete adsorption-desorption cycles with a reasonable recovery time by means of laser heating without the destruction of sensors' surface structures 34-36

The calculated adsorption energies corresponding to the most stable geometries are compiled in Fig 2. The computed adsorption energies indicate that pristine BlackP is less sensitive to both types of S-contained gases because of the low binding energies, amounting to 0.21 and $0.33 \mathrm{eV} /$ molecule for $\mathrm{H}_{2} \mathrm{~S}$ and $\mathrm{SO}_{2}$, respectively ${ }^{22}$. The $\mathrm{H}_{2} \mathrm{~S}$ molecule energetically adopts the vertical orientation where the $\mathrm{H}$ atoms direct towards the phosphorene surface (as shown in Fig. 3). This binding configuration resembles that reported in the previous work ${ }^{22}$. The shortest H-P and SP bond distances are 2.91 and $3.91 \AA$ and the H-S-H bond angle remains unaffected. Meanwhile, Fig. 4 reveals that the $\mathrm{SO}_{2}$ molecule prefers to be adsorbed on the hollow site with horizontal orientation pointing the $\mathrm{S}$ atom to bind to a phosphorus atom and the corresponding P-S bond distance is $3.00 \AA$. Like $\mathrm{H}_{2} \mathrm{~S}$, the O-S-O bond angle is unaltered. Note that the adsorption energy of $\mathrm{SO}_{2}$ is relatively higher than $\mathrm{H}_{2} \mathrm{~S}$ simply because the former directs $\mathrm{S}$ atom towards phosphorene and the difference in electronegativity between $\mathrm{S}$ and $\mathrm{P}$ is greater than that between $\mathrm{H}$ and $\mathrm{P}$. In addition, it is worth mentioning that the $\mathrm{H}-\mathrm{S}(\mathrm{S}-\mathrm{O})$ bond distance in an isolated $\mathrm{H}_{2} \mathrm{~S}\left(\mathrm{SO}_{2}\right)$ 
molecule is calculated to be 1.352 (1.455) $\AA$. After adsorption onto pure BlackP, H-S (S-O) bond length becomes 1.351 (1.456) A, resembling their original values. Apparently, the large bond lengths between the gas molecule and phosphorene and the negligible changes in the bond angles support the low binding energies. The findings indicate that these gases physisorbed on BlackP via weak vdW forces, resembling the weak adsorption on other elemental 2D materials, namely graphene nanoribbons $^{37}$, silicene ${ }^{38}$, and germanene ${ }^{25}$. Therefore, the generic elemental 2D materials in the pristine forms are chemically insensitive to $\mathrm{H}_{2} \mathrm{~S}$ and $\mathrm{SO}_{2}$. Note that the calculated binding energies are much greater than the thermal fluctuation at room temperature $(0.03 \mathrm{eV}$ at $300 \mathrm{~K})$. This means that the adsorption of both kinds of gases on the phosphorene surface is a stable process in which the target molecules remain attached to the sensor and the desorption cycles can be done by annealing at the elevated temperature.

On the other hand, the presence of impurities can enhance the adsorption energies, particularly for the case of the substitutional doping of $\mathrm{Fe}$ and $\mathrm{Mn}$ atoms. The adsorption energies of $\mathrm{H}_{2} \mathrm{~S}$ in $\mathrm{Fe}(\mathrm{Mn})$-doped BlackP are $1.02(0.81) \mathrm{eV} /$ molecule, around 4 times higher than that in the pristine one. The $\mathrm{H}_{2} \mathrm{~S}$ molecule is adsorbed on the top site with the horizontal orientation (as shown in Fig. 3) where the S atom is located above $\mathrm{Fe}(\mathrm{Mn})$ atom to form the $\mathrm{Fe}(\mathrm{Mn})-\mathrm{S}$ bond with the bond length of 2.34 (2.51) $\AA$, marginally shorter than in the case of pristine. Moreover, the adsorption energies of $\mathrm{SO}_{2}$ in $\mathrm{Fe}(\mathrm{Mn})$-doped BlackP, as depicted in Fig. 4, are 1.01 (0.96) $\mathrm{eV} / \mathrm{molecule}$, around 4 times higher than that in pristine. In addition, the adsorption of $\mathrm{H}_{2} \mathrm{~S}$ onto Fe- and Mn-doped BlackP results in insignificant changes in H-S distances, i. e., being 1.364 (1.355) $\AA$ in Mn-doped and 1.361 (1.357) in Fe-doped. However, there are increases in the $\mathrm{H}-\mathrm{S}-\mathrm{H}$ bond angle from the original value of $92.1^{\circ}$ to $93.5^{\circ}$ in Fe-doped and $92.9^{\circ}$ in Mn-doped. Unlike $\mathrm{H}_{2} \mathrm{~S}$, the $\mathrm{SO}_{2}$ molecule energetically favors the top side with the vertical orientation in which one $\mathrm{O}$ atom is directed $\mathrm{Fe}(\mathrm{Mn})$ atom. The $\mathrm{Fe}(\mathrm{Mn})-\mathrm{O}$ bond lengths are calculated to be 2.34 (2.51) $\AA$, also remarkably less than the pristine. The calculated bond lengths between the transition metals and the adsorbed molecules manifest that the chemical bonds are formed because they are in the same order with the combination of metallic radii of transition metals and the covalent radii of $\mathrm{S}(\mathrm{O})$ atom. These strong bonds are responsible for the enhanced adsorption energies. Furthermore, the adsorption of $\mathrm{SO}_{2}$ induces a notable 
elongation of $\mathrm{S}-\mathrm{O}$ because of the vertical arrangement of $\mathrm{SO}_{2}$ where one $\mathrm{O}$ atom is directed downward to BlackP. The corresponding S-O distances on Fe-doped and Mn-doped are 1.462 (1.532) $\AA$ and 1.463 (1.539) Å, respectively. Intriguingly, O-S-O bond angles are remarkably bent from the gaseous value of $120.0^{\circ}$ to $113.2^{\circ}$ in $\mathrm{Fe}$ doped and $112.9^{\circ}$ in Mn-doped.

Regarding the defects, the presence of M-vac and D-vac does not significantly intensify the adsorption energies, meaning that the occurrence of these defects plays an insignificant role in the detection sensitivity of these S-containing gases. Interestingly, Quad-vacant BlackP chemically binds to both kinds of gases with the appropriate adsorption energies. However, the adsorbed molecules get dissociated after adsorption which is not practically suitable for the reversible adsorptiondesorption process required for the sensing application.

The gas-binding energies can be qualitatively correlated with the sensitivity by using the statistical thermodynamic model to estimate the adsorption density ${ }^{\mathbf{3 9}, 40}$. The interaction between the solid sensor's surface and the adsorbed molecule along $z$ direction can be described by the Morse potential

$$
V(z)=D_{e}\left[e^{-2 \gamma\left(z-z_{e}\right)}-2 e^{-\gamma\left(z-z_{e}\right)}\right]
$$

where $D_{e}$ represents the depth of potential well (i.e., binding energy), $\gamma$ is the fitting parameter and $z_{e}$ refers to the equilibrium distance at which the binding energy is at the lowest as shown in Fig. 6. The gas molecule is attached to the sensor when the binding energy becomes negative. Here, the interaction between the gas molecules and the surface's sensor is assumed to be independent in planar $x-y$ plane. This is reasonable because the preferential binding site and orientation was exhaustively searched by sampling various sites and configurations prior to the use of Eq. (3). The eigenenergies of Schrödinger equation of the adsorbed gas molecule with Morse potential is

$$
E_{n}=\hbar \omega_{g}\left[\frac{[-2(2 n+1)+8 \Delta]^{2}}{64 \Delta}\right]
$$

where $\omega_{g}=\gamma\left(2 D_{e} / m_{g}\right)^{2}$ defines the characteristic frequency of the gas molecule of mass $m_{g}$ and $\Delta=D_{e} / \hbar \omega_{g}$. The gas adsorption density at the dilute concentration regime (i. e., ignoring the interaction between gas molecules) can be calculated form 
the number of adsorbed gas molecules $\left(n_{g}\right)$ per unit area $(A)$ according to the expression

$$
n_{g}=\frac{N}{A}=P_{g}\left[\frac{\lambda}{k_{B} T} \exp \left(\frac{D_{e}}{k_{B} T}\right) \sum_{n} \exp \left(-\frac{E_{n}}{k_{B} T}\right)\right]
$$

where the expression in the square bracket is Henry adsorption isotherm, $P_{g}$ stands for the partial pressure of the target gas and we assume it to be $1 \times 10^{-9}$ which corresponds to the dilute concentration limit. Fig. 6 shows the fitting of Morse potential of the gas molecules adsorbed on BlackP and the corresponding adsorption densities under the gas concentration of $1 \mathrm{ppb}$ (i. e. the partial pressure) at $300 \mathrm{~K}$. The densities of $\mathrm{H}_{2} \mathrm{~S}\left(\mathrm{SO}_{2}\right)$ on pristine, Fe-doped, and Mn-doped phosphorene are estimated to be $5.75 \times 10\left(1.67 \times 10^{7}\right), 5.87 \times 10^{30}\left(4.96 \times 10^{35}\right)$, and $4.26 \times 10^{23}(1.55$ $\left.\mathrm{x} 10^{35}\right) \mathrm{cm}^{-2}$, respectively. It is apparent that the adsorption densities of these Scontained gases on pristine BlackP are remarkably less than those on doped BlackP under the same conditions. This manifests that the latter case offers greater sensitivity to detecting these kinds of gases.

The ultra-sensitivities of doped BlackP are comprehensively attributed to the measurable changes in their electronic properties after gas exposure. One can further explore the comprehensive explanations by the analysis of electronic properties via the density of states (DOS) as depicted in Fig. 6. There is no change in the total DOS of pristine BlackP after being adsorbed by $\mathrm{H}_{2} \mathrm{~S}$ and $\mathrm{SO}_{2}$. This supports their low adsorption energies and manifests the weak vdW force between BlackP and the adsorbates. The weak vdW-type interaction cannot generate the substantial contrast in the electrical conductivity. It is possible to manipulate the sensitivity by means of surface functionalization by transition metals in order to intensify the charge transfer mechanism. The presence of transition-metal (TM) impurities results in the localized 3d-states near the Fermi level and the systems have become magnetic, see Fig. 6(b-c), as evident by the calculated magnetic moment of $\mathrm{Fe}$ and $\mathrm{Mn}$ as 1.40 and $2.80 \mu_{\mathrm{B}}$, respectively ${ }^{41}$. In principle, the electronic configurations of $\mathrm{Fe}$ and $\mathrm{Mn}$ are $[\mathrm{Ar}] 4 \mathrm{~s}^{2} 3 \mathrm{~d}^{6}$ and $[\operatorname{Ar}] 4 s^{2} 3 d^{5}$, respectively. Three electrons from the $3 d$-orbitals get involved in forming $\mathrm{Fe}(\mathrm{Mn})-\mathrm{P}$ bonds with three surrounding phosphorus atoms. This consequently leaves 1(2) unpaired electrons in the $d$ orbital which are intrinsically prone to incoming gas molecules. 
The adsorption of $\mathrm{SO}_{2}$ creates major changes in the total DOS of the TM doped BlackP, see Fig. 6(e-f). For the case of $\mathrm{SO}_{2}$ adsorption on to $\mathrm{Fe}(\mathrm{Mn})$-doped BlackP, there is an intensive overlap between $\mathrm{Fe}(\mathrm{Mn})-3 d$ and $\mathrm{O}-2 p$ states to form the strong $\mathrm{Fe}(\mathrm{Mn})-\mathrm{O}$ ionic bond which is a consequence of the charge transfer from $\mathrm{Fe}$ (Mn)-3d to O-2p (i.e. $\mathrm{P}$ being highly more electronegative). This transfer mechanism is supported by the graphical plot of charge density shown in Fig. 7 where there is charge depletion occurring at the proximity of TM atoms. On the other hand, the adsorption of $\mathrm{H}_{2} \mathrm{~S}$ produces the small changes in DOS of $\mathrm{Fe}(\mathrm{Mn})$-doped, despite the strong adsorption energies previously mentioned. In addition, there are negligible changes in the magnetic moments of $\mathrm{Fe}(\mathrm{Mn})$ in $\mathrm{Fe}(\mathrm{Mn})$-doped BlackP after $\mathrm{H}_{2} \mathrm{~S}$ and $\mathrm{SO}_{2}$ adsorption. However, these small changes are basically ascribed to the interaction between $\mathrm{O}-2 \mathrm{p}$ orbitals and $\mathrm{Fe}(\mathrm{Mn})-3 \mathrm{~d}$ orbitals which leads to spin cancelation. The reduction in magnetic moments is also manifested by the marked change in DOS of $\mathrm{Fe}(\mathrm{Mn})$-doped BlackP after adsorption. This indicates that Fe and Mn impurities do not significantly enhance the sensitivity of BlackP towards $\mathrm{H}_{2} \mathrm{~S}$ gas. Hence, it is concluded that substitutional doping of Fe to BlackP is a promising approach to enhance the sensitivity of BlackP towards $\mathrm{SO}_{2}$ detection. Another important parameter that describes the promise of efficient nanosensors is conductivity, which could be calculated by the following relation.

$$
\sigma=A \exp \left(-\mathrm{E}_{\mathrm{g}} / 2 \mathrm{k}_{\mathrm{B}} \mathrm{T}\right)
$$

Here $\sigma$ is electrical conductivity; $\mathrm{Eg}, \mathrm{k}_{\mathrm{B}}$ and $\mathrm{T}$ are the band gap, Boltzmann constant and temperature respectively. $\mathrm{A}$ is the constant of proportionality whose value depends on the system. ${ }^{42}$ It is apparent from above relation that the variation of energy gap would directly affect the conductivity of the sensor. Looking at the density of states plots in Fig. 6 one can hardly see any change in $E_{g}$ in case of pristine phosphorene. However a careful investigation confirms that both $\mathrm{Fe}$ and $\mathrm{Mn}$ functionalized phosphorene results into an apparent change in $\mathrm{E}_{\mathrm{g}}$ upon the exposure of even a single molecule of $\mathrm{SO}_{2}$. This conductivity change could be converted into an electric signal through external circuit. Thus foreign element functionalization enhances the sensing characteristics of phosphorene nanosheets.

\section{Conclusion}


In summary, we have performed first-principles DFT calculations coupled with the statistical analysis to unravel the structural, electronic, and gas sensing properties of pristine, defected, and metal substituted BlackP towards toxic $\mathrm{H}_{2} \mathrm{~S}$ and $\mathrm{SO}_{2}$ gas molecules. Our findings have revealed that pristine BlackP weakly interacts with both $\mathrm{H}_{2} \mathrm{~S}$ and $\mathrm{SO}_{2}$ by van der Waals (vdW) forces characterized by the small binding energies. The analysis of electronic properties via the density of states (DOS) indicates that there is a negligible change in DOS after gas exposure which confirms insensitive sensing. To intensity the binding energies, we have considered defects (mono-vacancy, di-vacancy, tri-vacancy and quad-vacancy) and substitutional impurities ( $\mathrm{Ti}, \mathrm{Si}, \mathrm{Mn}$ and $\mathrm{Fe}$ ) as the incentives. The presence of Mono and Di vacancies remains less energetically sensitive to both gas species because of the low adsorption energies. Meanwhile, tri and quad vacancies induce the dissociative adsorption, not suitable for the reversible adsorption-desorption cycles. Substitutional doping by $\mathrm{Fe}$ atoms is found to be a feasible approach to enhance the sensing resolution of $\mathrm{SO}_{2}$ detection because of the remarkable adsorption energy incorporated with the substantial variation in DOS after gas exposure. This modification in electronic properties is facilitated by the charge transfer mechanism from $\mathrm{Fe}-3 d$ to $\mathrm{P}$ $3 p$ which can generate the measurable electrical signal detect by the external circuit of the sensor. Our work highlights the functionality of BlackP for detecting toxic $\mathrm{SO}_{2}$ gas.

\section{Acknowledgements}

T. K. would like to acknowledge the Development and Promotion of Science and Technology Talent Project (DPST) for the financial support of this project (005/2559). The Nanotechnology Centre (NANOTEC), NSTDA Ministry of Science and Technology (Thailand) also supports T.K. through its program of Centre of Excellence Network, Integrated Nanotechnology Research Centre Khon Kaen University (Thailand). In addition, T. K. also acknowledges Prof. Udomsilp Pinsook from the department of Physics, Chulalongkorn University for providing the kind academic mentoring. We are grateful to Dr Hakkim Vovusha for his useful input in charge density calculations. T. H. and A. K. are indebted to the resources at NCI National Facility systems at the Australian National University through National Computational Merit Allocation Scheme supported by the Australian Government and 
the University of Queensland Research Computing Centre. A. K. acknowledges an Australian Research Council (ARC) Future Fellowship (FT170100373). 

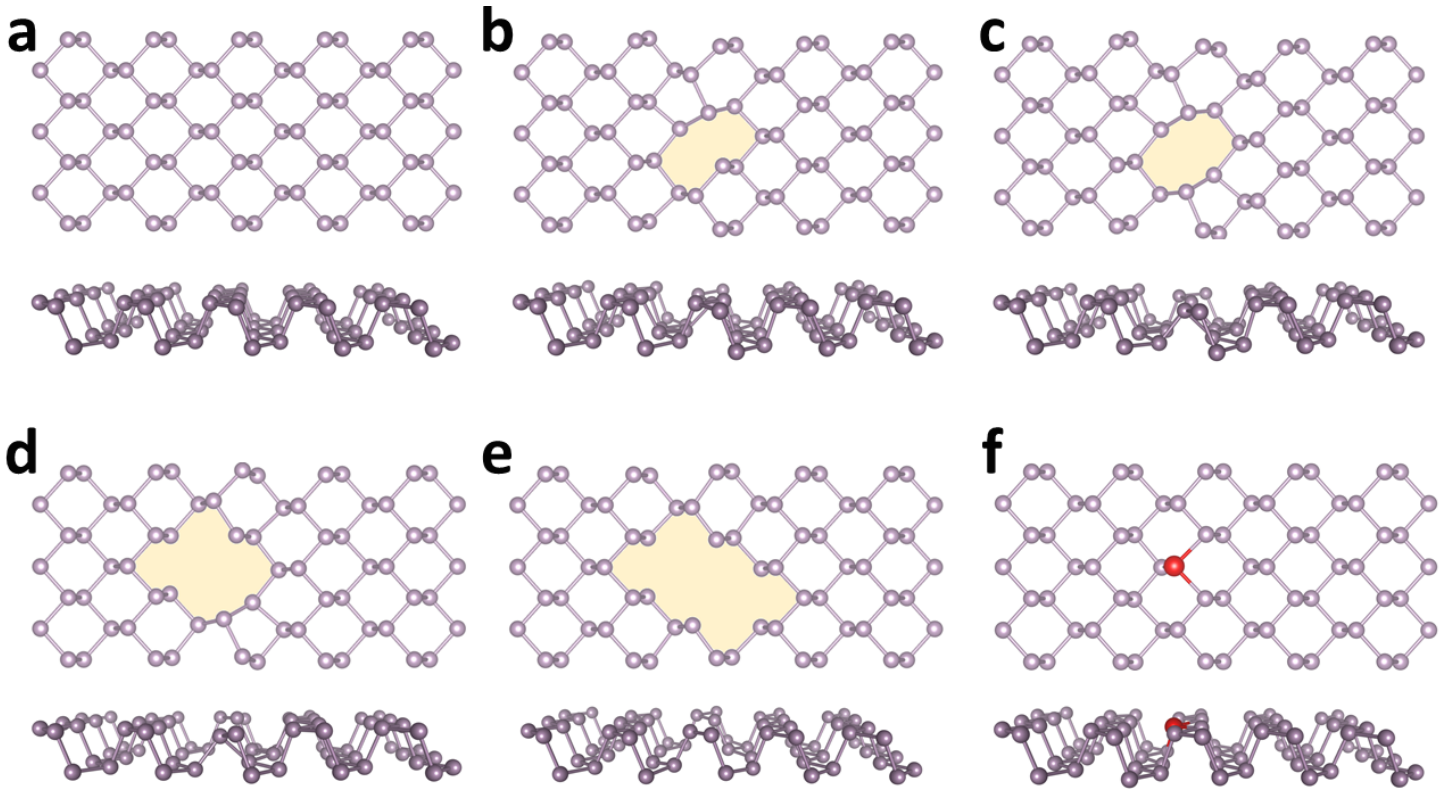

Fig. 1 The crystal structures (bird-eye and side views) of different kinds of phosphorene considered in this work (a) Pristine, (b) Mono-vacancy (M-vac), (c) Divacancy (D-vac), (d) Tri-vacancy (T-vac), (e) Quad-vacancy (Q-vac), and (f) Fe (Mn, $\mathrm{Si}, \mathrm{Ti})$-doped. Light purple and red spheres represent $\mathrm{P}$ and $\mathrm{Fe}(\mathrm{Mn}, \mathrm{Si}, \mathrm{Ti})$ atoms. 


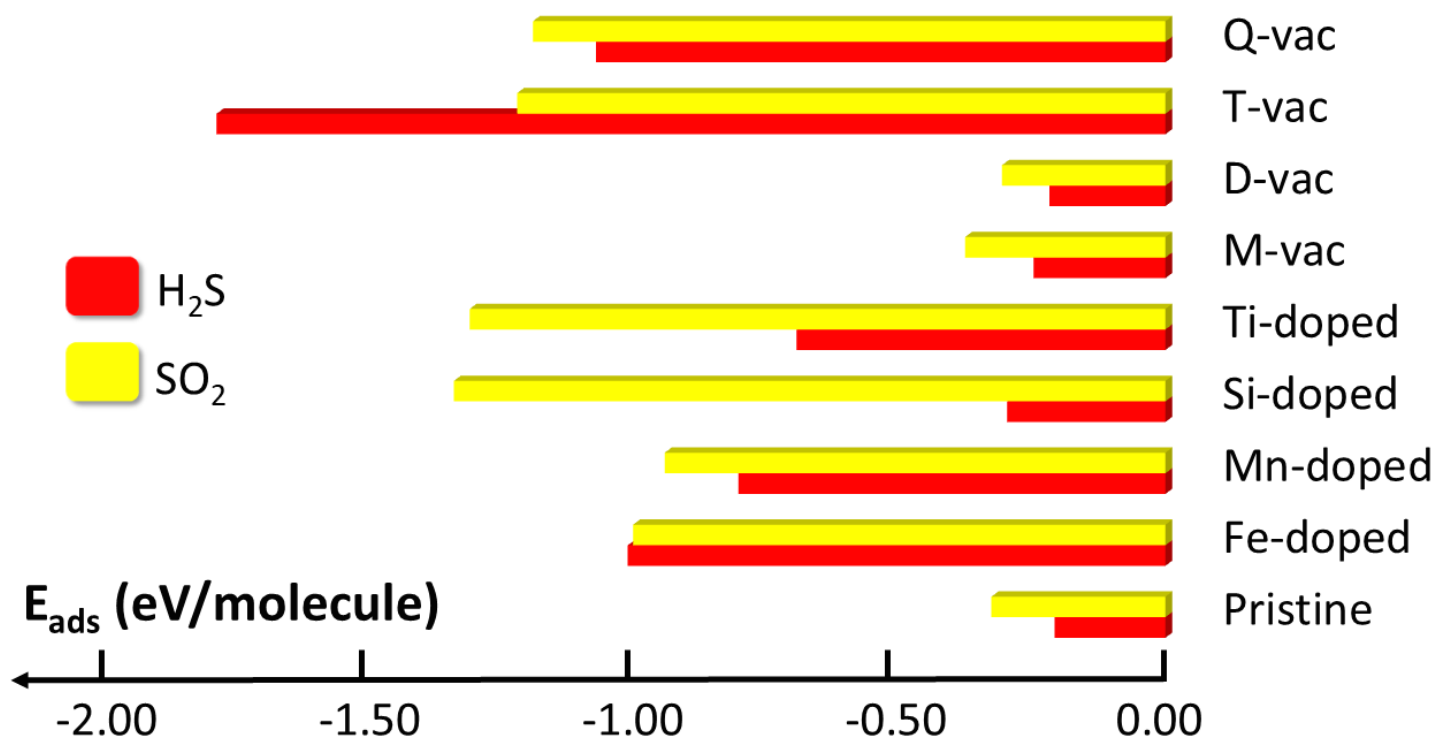

Fig 2. Adsorption energies of $\mathrm{H}_{2} \mathrm{~S}$ and $\mathrm{SO}_{2}$ gas molecules adsorbed on to several kinds of phosphorene. The capital D defines dissociative adsorption. 

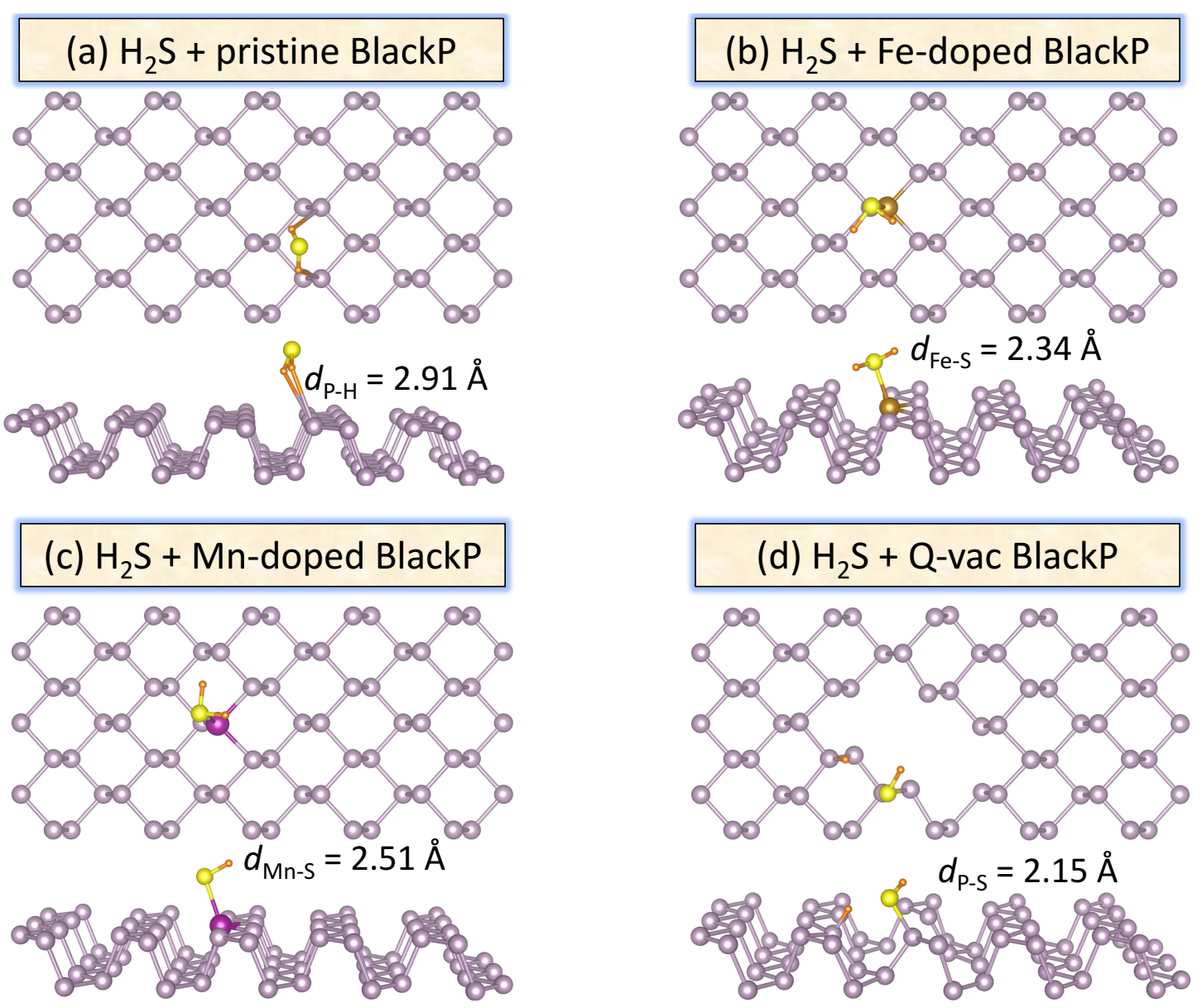

Fig 3. The crystal geometries (bird-eye and side views) of various types of phosphorene adsorbed by a $\mathrm{H}_{2} \mathrm{~S}$ molecule: (a) Pristine, (b) Fe-doped, (c) Mn-doped, and (d) Quad-vac. Yellow, gold, magenta, and orange balls denote S, Fe, Mn, and $\mathrm{H}$ atoms, respectively. 
(a) $\mathrm{SO}_{2}+$ pristine BlackP

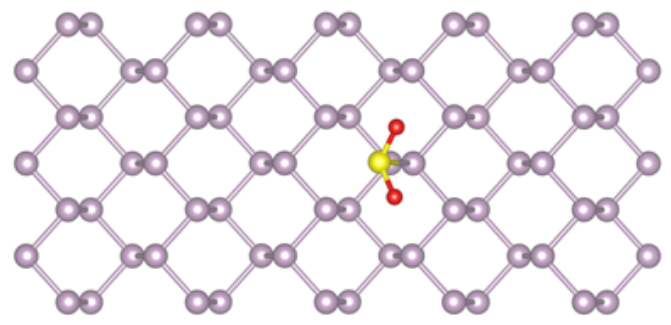

$\rho d_{\mathrm{p}-\mathrm{S}}=3.00 \AA$

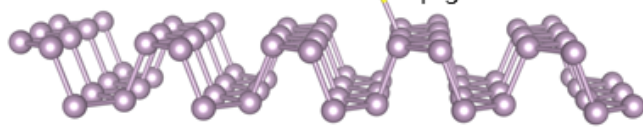

(c) $\mathrm{SO}_{2}+\mathrm{Mn}$-doped BlackP
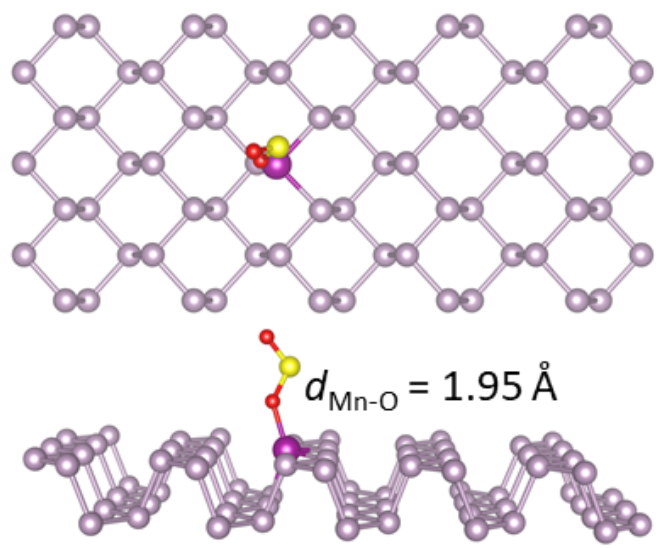

(b) $\mathrm{SO}_{2}+$ Fe-doped BlackP

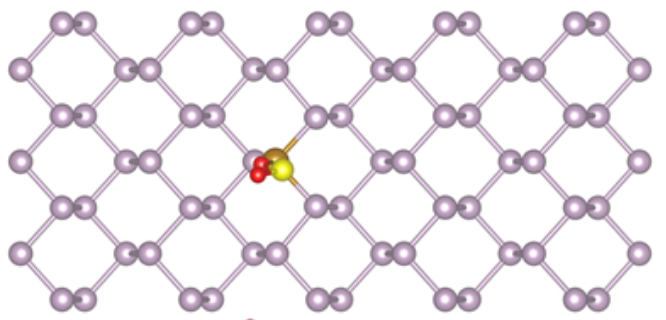

$d_{\mathrm{Fe}-\mathrm{O}}=1.90 \AA$

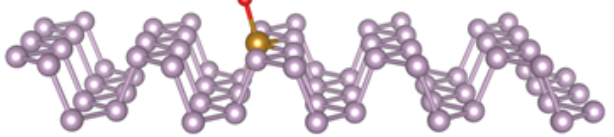

(d) $\mathrm{SO}_{2}+$ Q-vac BlackP

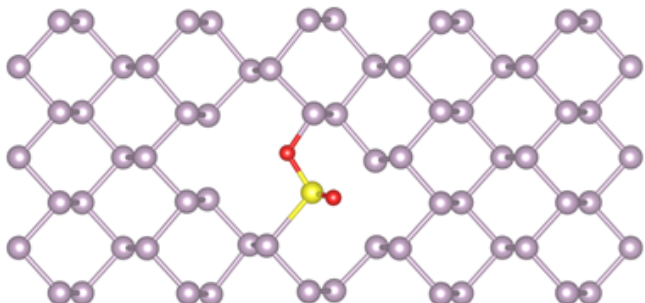

$$
d_{\mathrm{P}-\mathrm{S}}=2.55 \AA
$$

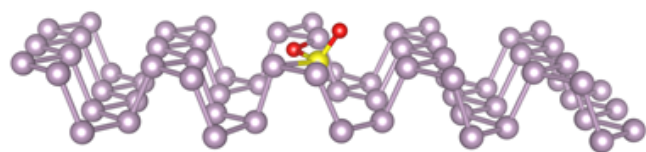

Fig 4. The crystal geometries (bird-eye and side views) of various types of phosphorene adsorbed by a $\mathrm{SO}_{2}$ molecule: (a) Pristine, (b) Fe-doped, (c) Mn-doped, and (d) Quad-vac. Yellow, gold, magenta, and red balls denote S, Fe, Mn, and O atoms, respectively. 

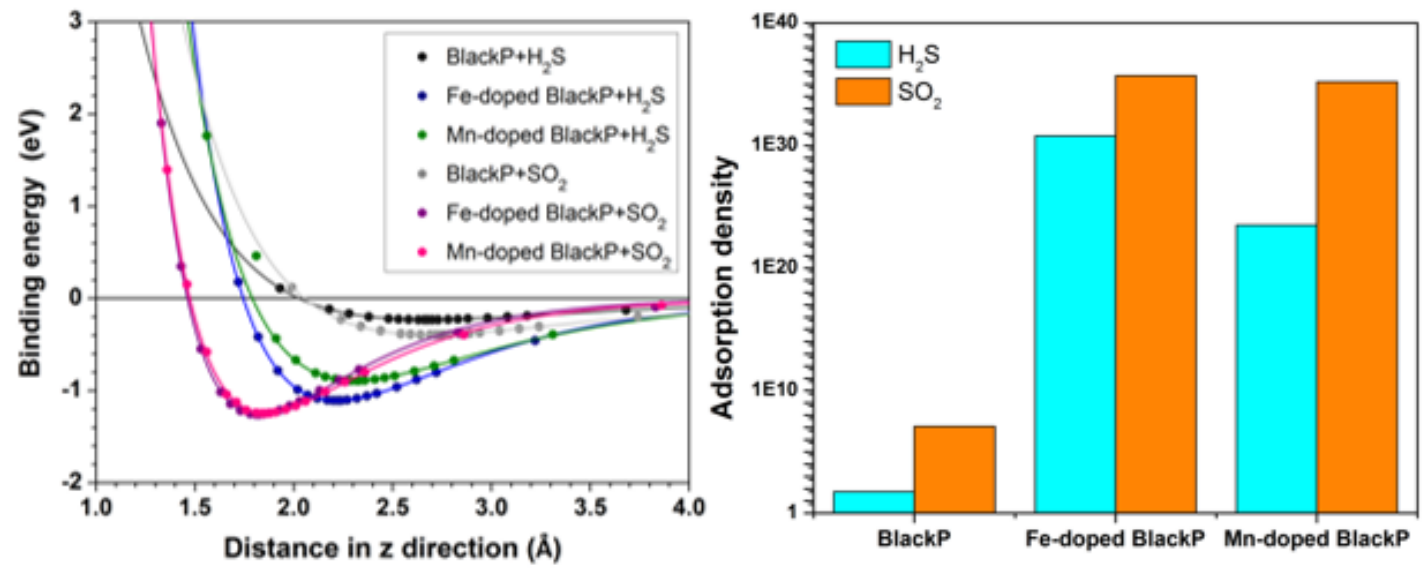

Fig. 5 (left) The Morse potential as a function of the distance between the BlackP surface and the gas molecule (i. e., the difference in the $z$ coordinate between the top $\mathrm{P}$ atom and the closest binding molecule as shown in Fig. 3 and 4) and (right) The adsorption densities of $\mathrm{H}_{2} \mathrm{~S}$ and $\mathrm{SO}_{2}$ molecules adsorbed on pure and doped BlackP under the gas concentration of $1 \mathrm{ppb}$ at $300 \mathrm{~K}$. 

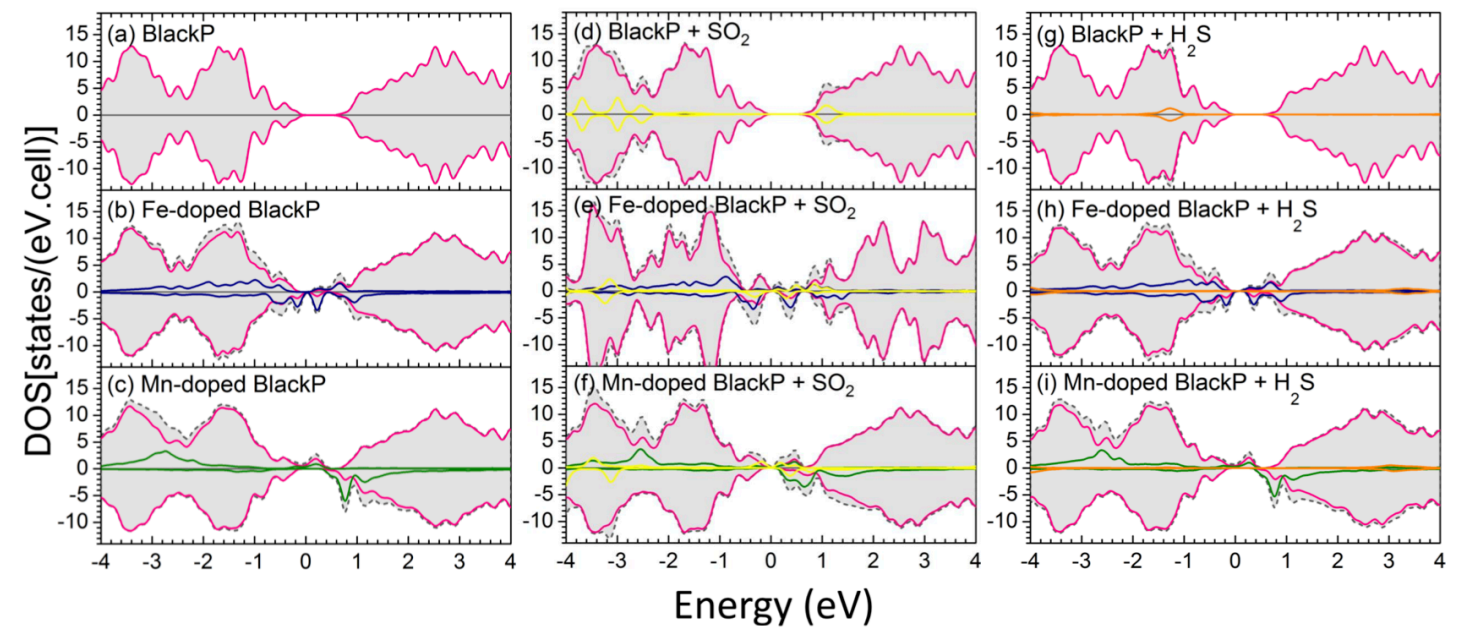

.........Total $P$ $\mathrm{Mn}$ $\mathrm{SO}_{2}$ $-\mathrm{H}_{2} \mathrm{~S}$

Fig. 6 Density of states (DOS) of phosphorene sheets adsorbed by $\mathrm{SO}_{2}$ and $\mathrm{H}_{2} \mathrm{~S}$ molecules. 

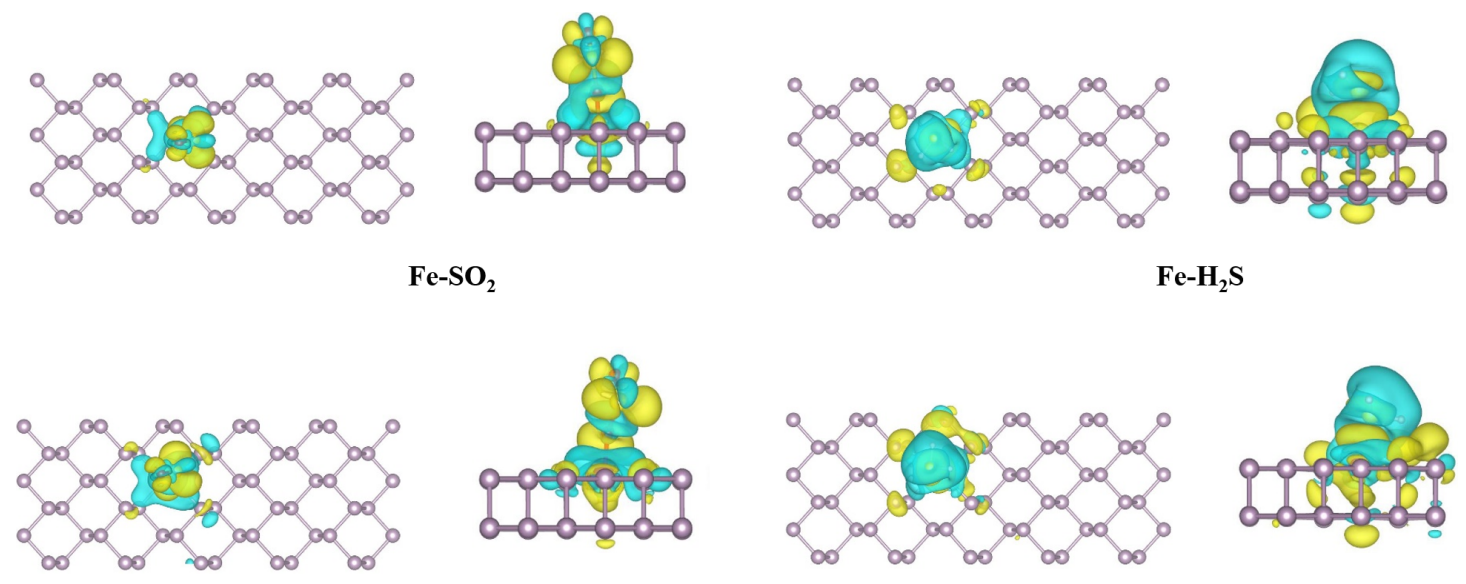

$\mathrm{Mn}-\mathrm{SO}_{2}$

$\mathrm{Mn}-\mathrm{H}_{2} \mathrm{~S}$

Fig. 7 Isosurface charge density of (a) $\mathrm{SO}_{2}+\mathrm{Fe}$-doped BlackP, (b) $\mathrm{H}_{2} \mathrm{~S}+\mathrm{Fe}$-doped BlackP, (c) $\mathrm{SO}_{2}+$ Mn-doped BlackP, and (d) $\mathrm{H}_{2} \mathrm{~S}+$ Mn-doped BlackP. Yellow and cyan colors represent the accumulation (-) and depletion (+) of electrons, respectively. The charge density is calculated by $n_{\text {gas }}+$ BlackP $-\left(n_{\text {BlackP }}+n_{\text {gas }}\right)$, where $n$ is charge density. The isosurface presented here is $0.005 \mathrm{e} / \AA^{3}$. 


\section{References}

1. Umar, A., Sensitivity, Selectivity, and Stability of Gas-Sensitive Metal-Oxide Nanostructures. Metal oxide Nano- structures and Their Applications, ed. Y.B. Hahn. 2010.

2. Schedin, F., Geim, A. K.; Morozov, S. V.; Hill, E. W.; Blake, P.; Katsnelson, M. I.; Novoselov, K. S. Detection of individual gas molecules adsorbed on graphene. Nat Mater, 2007, 6, 652-655.

3. Kong, J.; Franklin, N. R.; Zhou, C.; Chapline, M. G.; Peng, S.; Cho, K.; Dai, H. Nanotube Molecular Wires as Chemical Sensors. Science. 2000, 287, 622625.

4. Comini, E. Metal oxide nanowire chemical sensors: innovation and quality of life. Materials Today 2016, 19, 559 - 567.

5. Cho, S.Y., Lee, Y.; Koh, H-J.; Jung, H.; Kim, J-S.; Yoo, H-W.; Kim, J.; Jung, H-T. Superior Chemical Sensing Performance of Black Phosphorus: Comparison with MoS2 and Graphene. Adv Mater, 2016, 28, 7020-8.

6. Islam, M. S.; Hussain, T.; Rao, G. S.; Panigrahi, P.; Ahuja, R. Augmenting the sensing aptitude of hydrogenated graphene by crafting with defects and dopants. Sensors and Actuators B: Chemical 2016, 228, 317-321.

7. Rao, G. S.; Hussain, T.; Islam, M. S.; Sagynbaeva, M.; Gupta, D.; Panigrahi, P.; Ahuja, R. Adsorption mechanism of graphene-like $\mathrm{ZnO}$ monolayer towards $\mathrm{CO}_{2}$ molecules: enhanced $\mathrm{CO}_{2}$ capture. Nanotechnology 2016, 27, 015502-015508

8. Perkins, F.K.; Friedman, A. L.; Cobas, E.; Campbell, P. M.; Jernigan, G. G.; Jonker, B. T. Chemical vapor sensing with monolayer MoS2. NanoLett. 2013, 13, 668-73.

9. Kou, L., Du, A.; Cehn, C.; Frauenheim, T. Strain engineering of selective chemical adsorption on monolayer $\mathrm{MoS}_{2}$. Nanoscale 2014, 6, 5156-61.

10. Li, L., Yu, Y.; Ye, G. J.; Ge, Q.; Ou, X.; Wu, H.; Feng, D.; Chen, H. X.; Zhang, Y. Black phosphorus field-effect transistors. Nat Nanotechnol, 2014, 9, 372-377.

11. Liu, H.; Neal, A.T., Zhu, Z.; Luo, Z.; Xu, X.;Tomanek, D.; Ye, P. D. An Unexpected 2D Semiconductor with a high Hole Mobility. ACS Nano, 2014, 8, 4033 - 4041.

12. Reich, E.S. Phosphorene Excites Materials Scientists Nature, 2014. 506, 19.

13. Radisavljevic, B., Radenovic, A.; Brivio, J.; Giacometti, V.; Kis, A. Singlelayer MoS2 transistors. Nat Nanotechnol, 2011, 6, 147-50.

14. Castellanos-Gomez, A.; Vicarelli, L.; Prada, E.; Island, J. O.; NarasimhaAcharya, K. L.; Blanter, S. I.; Groenendijk, D. J.; Buscema, M.; Steele, G. A.; Alvarez, J. V.; Zandbergen, H. W.; Palacios, J. J.; van der Zant, S. J. Isolation and characterization of few-layer black phosphorus. 2D Materials, 2014, 1, 025001-025019. 
15. Xia, F.,Wang, H.; Jia, Y. Rediscovering black phosphorus as an anisotropic layered material for optoelectronics and electronics. Nat Commun, 2014, 5, 4458-4463.

16. Ray, S.J., First-principles study of MoS2, phosphorene and graphene based single electron transistor for gas sensing applications. Sensors and Actuators B: Chemical, 2016, 222, 492-498.

17. Abbas, A. N.; Liu, B.; Chen, L.; Ma, Y.; Cong, S.; Aroonyadet, N.; Kopf, M.; Nilges, T.; Zhou, C. Black Phosphorus Gas Sensor. ACS Nano, 2016, 9, 5618 $-5624$.

18. Wei, Q.; Peng, X. Superior mechanical flexibility of phosphorene and fewlayer black phosphorus. Applied Physics Letters, 2014, 104, 251915-251919.

19. Mahabal, M.; Deshpande, M. D.; Hussain, T.; Ahuja, R. Sensing Characteristics of Phosphorene Monolayers toward $\mathrm{PH}_{3}$ and $\mathrm{AsH}_{3}$ Gases upon the Introduction of Vacancy Defects. J. Phys. Chem. C 2016, 120, 20428-20436.

20. Lee, G., Kim, S.; Jung, S.; Jang, S.; Kim, J. Suspended black phosphorus nanosheet gas sensors. Sensors and Actuators B: Chemical, 2017, 250, 569573.

21. Cui, S., Pu, H.; Wells, S. A.; Wen, Z.; Mao, S.; Chang, J.; Hersam, M. C.; Chen, J. Ultrahigh sensitivity and layer-dependent sensing performance of phosphorene-based gas sensors. Nat Commun, 2015, 6, 8632-8640.

22. Yang, Q., Meng, R-S.; Jiang, J-K.; Liang, Q-H.; Tan, C-J.; Cai, M.; Sun, X.; Yang, D-G.; Ren, T-L.; Chen, X-P. First-Principles Study of Sulfur Dioxide Sensor Based on Phosphorenes. IEEE Electron Device Letters, 2016, 37, 660662.

23. Cho, S-Y., Kim, S. J.; Lee, Y.; Kim, J-S.; Jung, W-B.; Yoo, H-W.; Kim, J.; Jung, H-T. Highly Enhanced Gas Adsorption Properties in Vertically Aligned MoS2 Layers. ACS Nano, 2015, 9, 9314-21.

24. Sarkar, D., Xien, X.; Kang, J.; Zhang, H.; Liu, W.; Navarrete, J.; Moskovist, M.; Banerjee, K. Functionalization of transition metal dichalcogenides with metallic nanoparticles: implications for doping and gas-sensing. Nano Lett, 2015, 15, 2852-62.

25. Hussain, T., Kaewmaraya, T.; Chakraborty, S.; Vovusha, H.; Amornkitbamrung, V.; Ahuja, R. Defected and Functionalized Germanenebased Nanosensors under Sulfur Comprising Gas Exposure. ACS Sensors 2018, 3, 867-874.

26. G. Kresse, J.F., Efficient iterative schemes for ab initio total-energy calculations using a plane-wave basis set. Phys. Rev. B, 1996, 54, 1116911186.

27. Blochl, P.E., Projector augmented-wave method. Phys. Rev. B, 1994. 50, 17953-17979.

28. Perdew, J. P.; Burke, K.; Ernzerhof, M. Generalized Gradient Approximation Made Simple. Phys. Rev. Lett., 1996, 77, 3865-3868 
29. Grimme, S., Semiempirical GGA-type density functional constructed with a long-range dispersion correction. J Comput Chem. 2006, 27,1787-99.

30. Yu, M.; Trinkle, D. R. Accurate and efficient algorithm for Bader charge integration. J Chem Phys, 2011, 134, 064111-064118.

31. Qiao, J., Kong, X.; Hu, Z-X.; Yang, F.; Ji, W. High-mobility transport anisotropy and linear dichroism in few-layer black phosphorus. Nat Commun, 2014, 5, 4475-4481.

32. Li, X-B.; Guo, P.; Cao, T-F.; Liu, H.; Lau, W-M.; Liu, L-M. Structures, stabilities, and electronic properties of defects in monolayer black phosphorus. Scientic Reports, 2015, 5, 10848-10858.

33. Banhart, F.; Kotakoski, J.; Krasheninnikov, V. Structural Defects in graphene. ACS Nano 2011, 5, 26-41

34. Huang, B.; Li, Z.; Liu, Z.; Zhou, G.; Hao, S.; Wu, J.; Gu, B-L.; Duan, W. Adsorption of Gas Molecules on Graphene Nanoribbons and Its Implication for Nanoscale Molecule Sensor. J. Phys. Chem. C, 2008, 112, 13442-13446.

35. Hussain, T.; Hankel, M.; Searles, D. J. Improving Sensing of SulfurContaining Gas Molecules with ZnO Monolayers by Implanting Dopants and Defects. J. Phys. Chem. C 2017, 121, 24365-24375.

36. Abdulkader, T. S., Cui, X. Y.; Carter, D. J.; Rainger, S. P. Stampfl, C. Sensing sulfur-containing gases using titanium and tin decorated zigzag graphene nanoribbons from first-principles. Phys Chem Chem Phys, 2015, 17, 6925-6932.

37. Shao, L., Chen, G.; Ye, H.; Niu, H.; Wu, Y.; Zhu, Y.; Ding, B. Sulfur dioxide molecule sensors based on zigzag graphene nanoribbons with and without $\mathrm{Cr}$ dopant. Physics Letters A, 2014, 378, 667-671.

38. Hussain, T., Kaewmaraya, T.; Chakraborty, S.; Ahuja, R. Defect and Substitution-Induced Silicene Sensor to Probe Toxic Gases. The Journal of Physical Chemistry C, 2016, 120, 25256-25262.

39. Pu, H.H., Rhim, S. H.; Gajdardziksa-Josifovska, M.; Hirschmugl, C. J.; Weinert, M.; Chen, J. H. A statistical thermodynamics model for monolayer gas adsorption on graphene-based materials: implications for gas sensing applications. RSC Adv. 2014, 4, 47481-47487.

40. Li, P., Zhang, D.; Jiang, C.; Zong, X.; Cao, Y. Ultra-sensitive suspended atomically thin-layered black phosphorus mercury sensors. Biosens Bioelectron, 2017, 98, 68-75.

41. Hong, A.H.a.J., Transition Metal Doped Phosphorene: First-Principles Study. J Chem Phys, 2015, 119, 9198 - 9204.

42. Nagarajan, V.; Chandiramouli, R. Sensing properties of monolayer borophene nanosheet towards alcohol vapors: a first-principles study, J. Mol. Graphics Modell. 2017, 73, 208-216. 